\title{
Risk Factors for Infarct Growth in Patients with Acute Ischemic Stroke
}

Praneeta Konduri ${ }^{1,2}$, Amber Bucker ${ }^{1,2}$, Merel Boers ${ }^{1,2}$, Olvert Berkhemer ${ }^{1,2}$, Joost Bot ${ }^{3}$, Albert Yoo $^{4}$, Wim van Zwam ${ }^{5}$, Robert van Oostenbrugge ${ }^{6}$, Aad van der Lugt ${ }^{7}$, Diederik Dippel ${ }^{8}$, Yvo Roos ${ }^{9}$, Charles Majoie ${ }^{2}$, Henk Marquering ${ }^{1,2}$ ON BEHALF OF THE MRCLEAN INVESTIGATORS

${ }^{1}$ Biomedical Engineering and Physics, AMC, Netherlands; 2 Department of Radiology and Nuclear Medicine, AMC, Netherlands; ${ }^{3}$ Department of Radiology, VUmc, Netherlands; ${ }^{4}$ Department of Radiology, Texas Stroke Institute, Dallas; 5 Department of Radiology, Maastricht University Medical Center, Netherlands; ${ }^{6}$ Department of Neurology, Maastricht University Medical Center, Netherlands; ${ }^{7}$ Department of Radiology and Nuclear Medicine, Erasmus MC, Netherlands; ${ }^{8}$ Department of Public Health, Erasmus MC, Netherlands;

${ }^{9}$ Department of Neurology, AMC, Netherlands

\section{Introduction}

An increase in infarct lesion growth after treatment is associated with less favorable outcome in patients with acute ischemic stroke (AIS) $)^{1}$

Aim: To identify predictors of infarct growth after 24 hours of stroke onset.

\section{Materials and methods}

Two-hundred-twenty-six AIS patients with anterior proximal occlusions, with 24 hour and 1-week follow up NCCT scans were retrospectively selected from the MRCLEAN trial $^{2}$ database. Variables associated with absolute or relative infarct growth in univariate linear regression analysis $(\mathrm{p}<0.10)$ were selected for multivariate linear regression analysis.

\section{Results}

Table: Descriptive statistics of infarct volume and results of the multivariate linear regression analysis with absolute and relative infarct growth as dependent variables

\section{Median Infarct Volume}

\section{4 hours}

1 week

Parameter
42 (IQR: 21-95) $\mathrm{mL}$ 78 (IQR: 33-146) $\mathrm{mL}$

\begin{tabular}{l|l}
$\begin{array}{l}\text { Correlation } \\
\text { Coefficient }\end{array}$ & $\mathbf{p}$ - value \\
\hline
\end{tabular}

Absolute Infarct Growth

Systolic Blood Pressure $\quad 0.28$

0.02

Time to randomization

0.08

0.05

Infarct Volume (24 hrs)

0.10

0.02
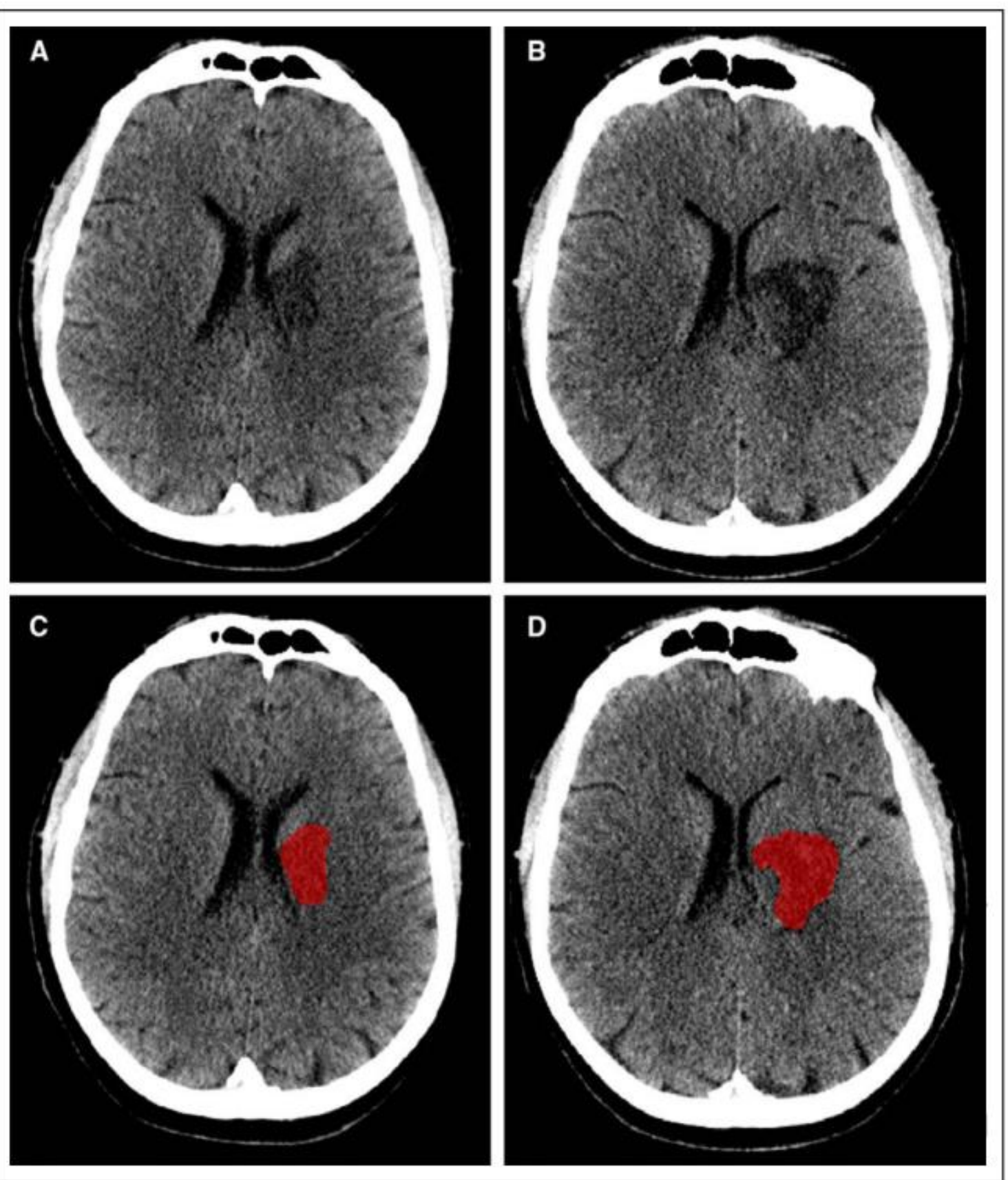

Figure: Example of ischemic lesion volume segmentation (represented in red) ${ }^{1}$.

A and C: 24-hr NCCT. B and D: 1 - week NCCT

\section{Conclusion}

Higher systolic blood pressure, longer time to randomization and larger initial infarct volume at 24 hours are independently associated with larger absolute infarct growth. Absence of myocardial infarction and smaller initial infarct volume are independently associated with relative infarct growth after 24 hours of stroke onset.

\section{References}

${ }^{1}$ Bucker, Amber, et al. Stroke 48.5 (2017):

1233-1240.

${ }^{2}$ Berkhemer, Olvert A., et al. New England Journal of Medicine 372.1 (2015): 11-20.
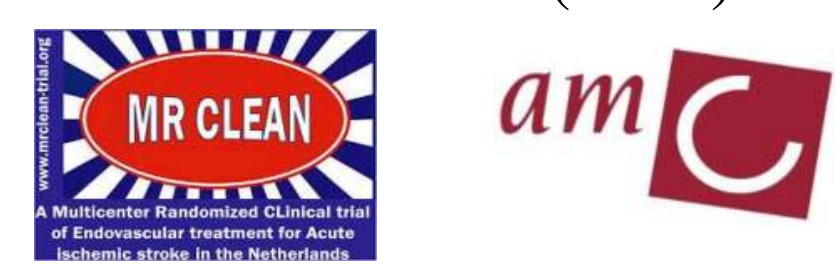

Texas Stroke Institute

\begin{tabular}{|l|l|l|}
\hline Myocardial Infarction & $-\mathbf{0 . 1 5}$ & $\mathbf{0 . 0 5}$ \\
\hline Infarct Volume (24 hrs) & $\mathbf{- 0 . 0 0 2}$ & $<0.01$ \\
\hline
\end{tabular}

